\title{
Application Chebyshev Polynomials for Determining the Eigenvalues of Sturm-Liouville Problem
}

\author{
DongYun Shen, Yong Huang* \\ Department of Mathematics, Foshan University, Foshan, Guangdong, China
}

Email address:

hyhy1223@126.com (Yong Huang)

To cite this article:

DongYun Shen, Yong Huang. Application Chebyshev Polynomials for Determining the Eigenvalues of Sturm-Liouville Problem. Applied and Computational Mathematics. Vol. 4, No. 5, 2015, pp. 369-373. doi: 10.11648/j.acm.20150405.16

\begin{abstract}
This paper discusses the eigenvalue problem of second-order Sturm-Liouville equation. We transform the governing differential equation to the Fredholm-Volterra integral equation with appropriate end supports. By expanding the unknown function into the shifted Chebyshev polynomials, we directly get the corresponding polynomial characteristic equations, where the lower and higher-order eigenvalues can be determined simultaneously from the multi-roots. Several examples of estimating eigenvalues are given. By comparison with the exact results in open literatures, the correctness and effectiveness of the present approach are verified.
\end{abstract}

Keywords: Sturm-Liouville Problem, Eigenvalues, Fredholm-Volterra Integral Equation, Chebyshev Polynomials

\section{Introduction}

Sturm-Liouville problems play an important role in several areas, such as physical, engineering and other scientific fields [1]-[2]. However, it is difficult to obtain exact expression of eigenvalues for such problem with different potential functions. In the past decades, various aspects of the numerical theory as well as approximate methods for this problem were presented to acquire the numerical results of eigenvalues. A simple asymptotic correction technique was employing by Andrew [3] to compute the eigenvalues of regular Sturm-Liouville problems with periodic or semiperiodic boundary conditions. Based on the boundary value methods, Ghelardnoi [4] used some linear multistep methods to discretize the Sturm-Liouville problem and investigate the approximations of eigenvalues. The eigenvalues of regular Sturm-Liouville problems with periodic boundary conditions was calculated in [5] by the finite difference scheme. In [6] and [7], Çelik and co-workers used the Chebyshev collocation method to investigated for the approximate computation of Sturm-Liouville eigenvalues by transforming the problems and given boundary conditions to matrix equation. Yuan et al. [8] proposed the Chebyshev collocation method to compute the approximate eigenvalues of regular Sturm-Liouville problems with two points and (semi-)periodic boundary conditions. Chen and Ma [9] used the Legendre-Galerkin-Chebyshev collocation method, which preserves the symmetry of the problem, to compute the approximate eigenvalues of the Sturm-Liouville problem with kinds of different boundary conditions. Zhang [10] cretized the Sturm-Liouville problems (SLPs) into standard matrix eigenvalue problems in order to achieve high accuracy and high efficiency by using the mapped barycentric Chebyshev differentiation matrix method. Based on the homotopy analysis method, Abbasbandy and Shirzadi [11] calculated the approximate eigenvalues of the second and fourth-order Sturm-Liouville problems. El-gamel and El-hady used the differential quadrature method and collocation method with sinc functions for computing eigenvalues of Sturm-Liouville problems [12].

In this paper, we will introduce an efficient approach to investigate the eigenvalues of Sturm-Liouville problem. For various boundary conditions, we transform the governing equation to the Fredholm-Volterra integral equations. Then a system of algebraic equations will be derived based on the Chebyshev polynomials expansion of the unknown function. The characteristic values can be easily determined from the existence condition of a nontrivial solution in the resulting system. Several examples used frequently in Sturm-Liouville problem will be used to demonstrate the accuracy of approximation.

\section{Integral Equation Method}

In the following, we consider the Liouville normal form of 
the general Sturm-Liouville equation:

$$
y^{\prime \prime}(x)+[\lambda-q(x)] y(x)=0, a \leq x \leq b,
$$

where the $q(x)$ is a given function, $y(x)$ is an unknown function satisfying certain boundary conditions, and the parameter $\lambda$ is the eigenvalue needed to be determined. During this paper, four familiar end conditions will be discussed:

$$
\begin{aligned}
& \text { Case A }: y(a)=y(b)=0, \\
& \text { Case B : } y(a)=y^{\prime}(b)=0, \\
& \text { Case C : } y^{\prime}(a)=y(b)=0, \\
& \text { Case D : } y(a)=-y(b), y^{\prime}(a)=-y^{\prime}(b) .
\end{aligned}
$$

The problem is actually of solving a set of second-order differential governing equation (1) and the corresponding end supports. Avoiding solving that differential equation directly, we introduce an integral equation method to convert the problem to Fredholm-Volterra integral equations with various boundary conditions. For this purpose, integrating both sides of Eq. (1) with respect to $x$ from $a$ to $x$, one gets

$$
y^{\prime}(x)+\int_{a}^{x}[\lambda-q(t)] y(t) d t=A_{1} .
$$

Then we repeat to integrate both sides of Eq. (6) with respect to $x$ from $a$ to $x$, yielding

$$
y(x)+\int_{a}^{x}(x-t)[\lambda-q(t)] y(t) d t=A_{2}+A_{1} x,
$$

where $A_{1}$ and $A_{2}$ are unknown constants that can be determined from the given boundary conditions. Once these two constants $A_{j}(j=1,2)$ can be uniquely obtained, we then substitute these values $A_{j}$ into Eq. (7) and immediately derive an integral equation in $y(x)$ of the following form:

$$
y(x)+\int_{a}^{x} K_{1}(x, t) y(t) d t+\int_{a}^{b} K_{2}(x, t) y(t) d t=0 .
$$

(1) Case A: $\quad y(a)=y(b)=0$

By setting $x=0,1$ in (7), respectively, we can get two linear equations about $A_{1}$ and $A_{2}$ as:

$$
\begin{gathered}
A_{2}+A_{1} a=0 \\
A_{2}+A_{1} b=\int_{a}^{b}(b-t)[\lambda-q(t)] y(t) d t .
\end{gathered}
$$

Solving the above algebraic equations, one can obtain

$$
\begin{aligned}
& A_{1}=\frac{1}{b-a} \int_{a}^{b}(b-t)[\lambda-q(t)] y(t) d t, \\
& A_{2}=\frac{-a}{b-a} \int_{a}^{b}(b-t)[\lambda-q(t)] y(t) d t .
\end{aligned}
$$

Substituting them back into (7), and after collection we get a Fredholm-Volterra integral equation as follows:

$$
y(x)+\int_{a}^{x} K_{1}(x, t) y(t) d t+\int_{a}^{b} K_{2}(x, t) y(t) d t=0
$$

with

$$
\begin{aligned}
& K_{1}(x, t)=(x-t)[\lambda-q(t)] \\
& K_{2}(x, t)=\frac{a-x}{b-a}(b-t)[\lambda-q(t)]
\end{aligned}
$$

(2) Case B:. $y(a)=y^{\prime}(b)=0$

Applying the condition $y(a)=0$ in (7) leads to

$$
A_{1} a+A_{2}=0
$$

On the other hand, we substitute the condition $y^{\prime}(b)=0$ to (6), yielding

$$
A_{1}=\int_{a}^{b}[\lambda-q(t)] y(t) d t
$$

Putting the above into Eq. (16), one gets

$$
A_{2}=-a \int_{a}^{b}[\lambda-q(t)] y(t) d t
$$

Finally, substituting $A_{j}$ into Eq. (7), we obtain a Fredholm-Volterra integral equation for case B as follows:

$$
y(x)+\int_{a}^{x} K_{1}(x, t) y(t) d t+\int_{a}^{b} K_{2}(x, t) y(t) d t=0
$$

with

$$
\begin{aligned}
& K_{1}(x, t)=(x-t)[\lambda-q(t)], \\
& K_{2}(x, t)=(a-x)[\lambda-q(t)] .
\end{aligned}
$$

(3) Case C:. $y^{\prime}(a)=y(b)=0$

Bearing $y^{\prime}(a)=0$ in mind, setting $x=a$ in (6) leads to

$$
A_{1}=0
$$

Applying the condition $y(b)=0$ to (7), we get 


$$
A_{2}=\int_{a}^{b}(b-t)[\lambda-q(t)] y(t) d t
$$

Plugged $A_{j}$ into Eq. (7), the final Fredholm-Volterra integral equation is derived as follows:

$$
y(x)+\int_{a}^{x} K_{1}(x, t) y(t) d t+\int_{a}^{b} K_{2}(x, t) y(t) d t=0,
$$

with

$$
\begin{aligned}
& K_{1}(x, t)=(x-t)[\lambda-q(t)] \\
& K_{2}(x, t)=(t-b)[\lambda-q(t)] .
\end{aligned}
$$

(4) Case D: $\quad y(a)=-y(b), \quad y^{\prime}(a)=-y^{\prime}(b)$

Setting $x=a, b$ in (6) and (7), respectively, after using the condition $y^{\prime}(a)=-y^{\prime}(b)$ and $y(a)=-y(b)$, we can obtain

$$
\begin{gathered}
2 A_{1}=\int_{a}^{b}[\lambda-q(t)] y(t) d t \\
2 A_{2}+A_{1}(a+b)=\int_{a}^{b}(b-t)[\lambda-q(t)] y(t) d t .
\end{gathered}
$$

Therefore, $A_{1}$ and $A_{2}$ can be obtained by solving the above algebraic equations

$$
\begin{gathered}
A_{1}=\int_{a}^{b} \frac{\lambda-q(t)}{2} y(t) d t \\
A_{2}=\int_{a}^{b} \frac{b-a-2 t}{4}[\lambda-q(t)] y(t) d t .
\end{gathered}
$$

With these obtained $A_{j}$, after some simplification we finally derive the Fredholm-Volterra integral equation as follows:

$$
y(x)+\int_{a}^{x} K_{1}(x, t) y(t) d t+\int_{a}^{b} K_{2}(x, t) y(t) d t=0,
$$

with

$$
\begin{gathered}
K_{1}(x, t)=(x-t)[\lambda-q(t)] \\
K_{2}(x, t)=\frac{a+2 t-b-2 x}{4}[\lambda-q(t)] .
\end{gathered}
$$

\section{Characteristic Equation of the Problem}

In the preceding section, for some typical boundary conditions, we have converted the governing differential equation (1) to the corresponding Fredholm-Volterra integral equation (8). In the following, a simple approach will be introduced to solve the integral equations. It is well-known that the first kind of Chebyshev polynomials can be derived by the following recurrence relations:

$$
\begin{aligned}
& T_{0}^{*}(\xi)=1, \quad T_{1}^{*}(\xi)=\xi \\
& T_{i+1}^{*}(\xi)=2 \xi T_{i}^{*}(\xi)-T_{i-1}^{*}(\xi),
\end{aligned}
$$

where $\xi$ is over the interval $[-1,1]$. After introducing a variable substitution $x=\frac{b-a}{2} \xi+\frac{a+b}{2}$, we can easily derive the Chebyshev polynomials over the interval $[a, b]$

$$
\begin{gathered}
T_{0}(x)=1, \quad T_{1}(x)=\frac{2 x-a-b}{b-a}, \\
T_{i+1}(x)=\frac{4 x-2 a-2 b}{b-a} T_{i}(x)-T_{i-1}(x) .
\end{gathered}
$$

Firstly, we expand the unknown $y(x)$ into the shifted Chebyshev polynomials in general over the interval $[a, b]$ as

$$
y(x)=\sum_{i=0}^{N} c_{i} T_{i}(x), \quad a \leq x \leq b
$$

where $c_{i}$ are unknown coefficients, and $N$ is a certain positive integer. Putting the expansion (38) into the resulting Fredholm-Volterra integral Eq. (8) for each case leads to

$$
\begin{aligned}
& \sum_{i=0}^{N} c_{i} T_{i}(x)+\sum_{i=0}^{N} c_{i} \int_{a}^{x} K_{1}(x, t) T_{i}(t) d t \\
& +\sum_{i=0}^{N} c_{i} \int_{a}^{b} K_{2}(x, t) T_{i}(t) d t=0 .
\end{aligned}
$$

Multiplying both sides of (39) by $T_{j}(x)$ and then integrating with respect to $x$ between $a$ and $b$, one can get a system of linear algebraic equations in $c_{i}$ :

$$
\sum_{i=0}^{N}\left(T_{i j}+K_{1 i j}+K_{2 i j}\right) c_{i}=0, \quad j=0,1,2, . . N
$$

with

$$
\begin{aligned}
& T_{i j}=\int_{a}^{b} T_{i}(x) T_{j}(x) d x, K_{1 i j} \\
& =\int_{a}^{b} \int_{a}^{x} K_{1}(x, t) T_{i}(t) T_{j}(x) d t d x, K_{2 i j} \\
& =\int_{a}^{b} \int_{a}^{b} K_{2}(x, t) T_{i}(t) T_{j}(x) d t d x
\end{aligned}
$$

In order to acquire a nontrivial solution of the linear algebraic equations (40), the determinant of the coefficient matrix of the system has to vanish, namely:

$$
\operatorname{det}\left(T_{i j}+K_{1 i j}+K_{2 i j}\right)=0
$$


Therefore, we have derived the characteristic equation in eigenvalue $\lambda$. By inspecting this obtained equation, we can easily find that it is actually a polynomial in eigenvalue $\lambda$ that has multi-roots of positive solutions, which are corresponding to the lower and higher eigenvalues.

\section{Illustrative Examples}

In this section, several illustrative examples used frequently in Sturm-Liouville problem are presented to show the efficiency of the proposed method. We first consider the following example

$$
-y^{\prime \prime}(x)=\lambda y(x)
$$

where $q(x)=0$. This is a second-order ordinary differential equation. A general solution to Eq. (43) can be immediately obtained based on a standard approach for solving the above equation

$$
y=C_{1} \cos (\sqrt{\lambda} x)+C_{2} \sin (\sqrt{\lambda} x)
$$

where $C_{1}$ and $C_{2}$ are unknown constants that can be determined from the boundary conditions. Here three boundary conditions are discussed: (1) $y(0)=y(1)=0 ;(2)$ $y(0)=y^{\prime}(1)=0 ; \quad$ (3) $y^{\prime}(0)=y(1)=0$. Substituting the Eq. (44) to the corresponding supported ends, after collection we can get the exact characteristic values of (43): (1) $\lambda=(k \pi)^{2}$;

(2) $\lambda=\left(k \pi-\frac{1}{2} \pi\right)^{2} ;$ (3) $\lambda=\left(k \pi-\frac{1}{2} \pi\right)^{2}, \quad k \in Z^{+}$. In order to check the correctness and convergence of the introduced approach, we have calculated the first four characteristic values of Sturm-Liouville equation (43) by taking different $N$ values in (38). The evaluated results and the exact characteristic values $\lambda$ are listed in Table 1 for the condition $y(0)=y(1)=0$. With $N$ increasing from 6 to 12 , the errors between the numerical and exact results dramatically decrease. This indicates that the numerical results have a rapid convergence with $N$ increasing. From Table 1 we can find that the obtained results of $N=12$ are in excellent agreement with exact ones, which are identical to each other up to 10 decimal digits for the first two eigenvalues Based on the present approach, we have calculated first four characteristic values $\lambda$ for Case 2 and Case 3 with $N=12$ where the results and the relative errors between those are tabulated in Table 2. A good agreement between the present computed results and exact results can be observed for the two cases from Table 2. Sturm-Liouville value problem arises in many physical, engineering and other scientific fields. The most important characteristic value for such problem is the first-order value, which is corresponding to the fundamental natural frequency or critical buckling loads or other material properties in those fields. We can find that our results of the first-order only letting $N=8$ are identical to the exact results.

Table 1. Numerical and exact results for Example 1 with $y(0)=y(1)=0$

\begin{tabular}{llllll}
\hline$k$ & $N=6$ & $N=8$ & $N=10$ & $N=12$ & Exact solution \\
\hline 1 & 9.8696044014 & 9.8696044011 & 9.8696044011 & 9.8696044011 \\
2 & 39.4797374430 & 39.4784194279 & 39.4784176054 & 39.4784176044 \\
3 & 88.8637975768 & 88.8266312672 & 88.8264400203 & 88.8264396102 \\
4 & 167.9171940870 & 158.2551516722 & 157.9179860576 & 157.9136936606 \\
\hline
\end{tabular}

\begin{tabular}{|c|c|c|c|c|c|c|}
\hline & $y(0)=y^{\prime}(1)=0$ & & & $y^{\prime}(1)=y(0)=0$ & & \\
\hline$k$ & Exact solution & Present & Relative errors & Exact solution & Present & Relative errors \\
\hline 1 & 2.4674011003 & 2.4674011003 & 0 & 2.4674011003 & 2.4674011003 & 0 \\
\hline 3 & 61.6850275068 & 61.6850275069 & $1.62 \times 10^{-12}$ & 61.6850275068 & 61.6850275069 & $1.62 \times 10^{-12}$ \\
\hline 4 & 120.9026539133 & 120.9026543279 & $3.42 \times 10^{-9}$ & 120.9026539133 & 120.9026543279 & $3.42 \times 10^{-9}$ \\
\hline
\end{tabular}

Table 2. Numerical and exact characteristic values for Example 1 with $N=12$.

Next we consider the following Sturm-Liouville problem

$$
-y^{\prime \prime}(x)+10 \cos (2 x) y(x)=\lambda y(x),
$$

with the boundary condtion $y\left(-\frac{\pi}{2}\right)=-y\left(\frac{\pi}{2}\right), y^{\prime}\left(-\frac{\pi}{2}\right)=-y^{\prime}\left(\frac{\pi}{2}\right)$. By using the mapped barycentric Chebyshev differentiation matrix method, Zhang [10] has calculated the characteristic values $\lambda$ of sucn Sturm-Liouville problem. For $N=12$, we employ the introduced method to determine the first four eigenvalues' approximations. The relative errors between the exact solution and our numerical results are tabulated in Table 3. Table 3 demonstrates that the present results are in very good agreement with the exact results [10].
Table 3. Numerical results of eigenvalues and the relative errors with $N=12$

\begin{tabular}{llll}
\hline$k$ & Exact results[10] & Present results $(N=12)$ & $\begin{array}{l}\text { Relative } \\
\text { errors }\end{array}$ \\
1 & 1.8581875415 & 1.8581900975 & $1.37 \times 10^{-6}$ \\
2 & 9.2363277137 & 9.2364554543 & $1.38 \times 10^{-5}$ \\
3 & 11.5488320363 & 11.5488428274 & $9.34 \times 10^{-7}$ \\
4 & 25.5108160463 & 25.5136131113 & $1.10 \times 10^{-4}$ \\
\hline
\end{tabular}

As the last example, we discuss the following Sturm-Liouville problem

$$
-y^{\prime \prime}(x)+e^{x} y(x)=\lambda y(x),
$$


with conditions (1) $\quad y(0)=y(\pi)=0 \quad$ and $\quad$ (2) $y(0)=y^{\prime}(\pi)=0$. The uniderivative Simpson method (USM)[8] and and Numerov's method (NM) [13] have been used to solve this Sturm-Liouville problem. The numerical results calculated by the Eq. (42), and the relative errors between the present results with $N=12$ and the exact results are listed in the Table 4 for two cases. Our results of $N=12$ coincide well with the exact solutions.

Table 4. Numerical results of eigenvalues and the relative errors with $N=12$.

\begin{tabular}{llllll}
\hline$k$ & $y(0)=y(\pi)=0$ & & \multicolumn{3}{c}{$y(0)=y^{\prime}(\pi)=0$} \\
\hline & Exact [8] & Present & Relative errors & Exact [13] & Present \\
\hline 1 & 4.8966694 & 4.8966693800 & $4.08 \times 10^{-9}$ & 4.89571 & 4.8957132596 \\
2 & 10.045190 & 10.0451899007 & $9.89 \times 10^{-9}$ & 9.99955 & 9.9995498471 \\
3 & 16.019267 & 16.0192679511 & $5.94 \times 10^{-8}$ & 15.4685 & 15.4685140327 \\
4 & 23.266271 & 23.2662808698 & $4.24 \times 10^{-7}$ & 21.0371 & 21.0371567754 \\
\hline
\end{tabular}

\section{Conclusions}

This paper presented a simple and efficient method to determine the eigenvalues of the second-order Sturm-Liouville problem. Instead of directly solving the differential equation, we transform the governing equation to the corresponding Fredholm-Volterra integral equations with kinds of boundary conditions. By expanding the unknown functions into the shifted Chebyshev polynomials, a system of linear algebraic equations will be obtained, where the lower and higher eigenvalues can be effectively computed from the characteristic polynomial equations. Compared our results with the exact solutions, the accuracy and effectiveness of the introduced method have been confirmed.

\section{Acknowledgements}

DongYun Shen appreciates the support from the special funds on science and technology innovation for Guangdong college students.

\section{References}

[1] MA Al-Gwaiz, Sturm-Liouville Theory and its Applications, Springer-Verlag, London, 2008.

[2] Veerle Ledoux, Study of special algorithms for solving Sturm-Liouville and Schrödinger equations. Ph.D Thesis, Department of Applied Mathematics and Computer Science, Ghent University, 2007.

[3] Alan L Andrew, Correction of finite difference eigenvalues of periodic Sturm-Liouville problems, J. Austral. Math. Soc. Ser. B 30 (1989) 460-469.
[4] Paolo Ghelardoni, Approximations of Sturm--Liouville eigenvalues using boundary value methods, Appl. Numer. Math. 23 (1997) 311--325.

[5] DJ Condon, Corrected finite difference eigenvalues of periodic Sturm-Liouville problems, Appl. Numer. Math. 30 (1999) 393-401.

[6] İbrahim Çelik, Approximate computation of eigenvalues with Chebyshev collocation method, Appl. Math. Comput. 168 (2005) 125-134.

[7] İbrahim Çelik, Guzin Gokmen, Approximate solution of periodic Sturm-Liouville problems with Chebyshev collocation method, Appl. Math. Comput. 170 (2005) 285-295.

[8] Quan Yuan, Zhiqing He, Huinan Leng, An improvement for Chebyshev collocation method in solving certain Sturm-Liouville problems, Appl. Math. Comput. 195 (2008) 440-447.

[9] Lan Chen, He-Ping Ma, Approximate solution of the Sturm-Liouville problems with Legendre-Galerkin-Chebyshev collocation method, Appl. Math. Comput. 206 (2008) 748-754.

[10] Xuecang Zhang, Mapped barycentric Chebyshev differentiation matrix method for the solution of regular Sturm-Liouville problems, Appl. Math. Comput. 217 (2010) 2266-2276.

[11] Saeid Abbasbandy, A new application of the homotopy analysis method: Solving the Sturm-Liouville problems, Commun. Nonlinear Sci. Numer. Simulat. 16 (2011) 112-126.

[12] Mohamed El-gamel, Mahmoud Abd El-hady, Two very accurate and efficient methods for computing eigenvalues of Sturm-Liouville problems, Appl. Math. Modell. 37 (2013) 5039-5046.

[13] Alan L Andrew, Twenty years of asymptotic correction for eigenvalue computation, ANZIAM J. 42 (2000) C96-C116. 